\title{
EGFR-TKI-sensitive mutations in lung carcinomas: are they related to clinical features and CT findings?
}

This article was published in the following Dove Press journal:

Cancer Management and Research

\section{Xiaoyi Qin' \\ Xiaolong $\mathrm{Gu}^{2}$ \\ Yingru $\mathrm{Lu}^{3}$ \\ Wei Zhou ${ }^{3}$}

'Department of Hematology, The First Affiliated Hospital of Wenzhou Medical University, Wenzhou,

Zhejiang, People's Republic of China; ${ }^{2}$ Department of Pneumology, Ningbo Yinzhou No. 2 Hospital, Ningbo, Zhejiang, People's Republic of China; ${ }^{3}$ Department of Intensive Care Unit, The First Affiliated Hospital of Wenzhou Medical University, Wenzhou, Zhejiang, People's Republic of China
Correspondence: Wei Zhou Department of Intensive Care Unit, The First Affiliated Hospital of Wenzhou Medical University, Nan Bai Xiang Street, Ouhai District, Wenzhou, Zhejiang 325000, People's Republic of China Tel +86I39588759I6 Emailwyyyzw@yahoo.com
Background: Epidermal growth factor receptor (EGFR) mutation testing is restricted to several limitations. In this study, we examined the relationship between EGFR mutation status and clinicoradiological characteristics in a Chinese cohort of patients.

Materials and methods: The data of patients who were diagnosed with lung carcinoma and underwent both EGFR testing and chest computed tomography (CT) at our hospital between January 1, 2011, and November 31, 2015, were retrospectively analyzed. The age, sex, and smoking index of the patients, the size, margin, and density of the tumor, and the presence of specific signs visible on the CT images were assessed.

Results: The results showed a higher rate of EGFR-tyrosine kinase inhibitor (TKI)-sensitive group than nonsensitive group in female patients and patients with a low smoking index $(P<0.001$, both). In logistic regression analyses, tumor size $(P<0.001)$, smooth margins $(P=0.015)$, and angular margins $(P<0.001)$ were independent negative predictors of EGFR-TKI-sensitive group. Pleural indentation $(P<0.001)$ and air bronchogram $(P=0.025)$ were independent positive predictors of EGFR-TKI-sensitive group. Patients with squamous cell carcinoma had fewer sensitive mutations than those with either adenocarcinoma $(P<0.001)$ or adenosquamous carcinoma $(P<0.001)$. Conclusion: Clinical and CT characteristics differed significantly between EGFR-TKI-sensitive and nonsensitive groups. Our findings may be useful in deciding therapeutic strategies for patients in whom EGFR testing is not possible.

Keywords: chest computed tomography, epidermal growth factor receptor, ground-glass opacity, lung carcinomas, mutations

\section{Introduction}

Lung cancer remains the leading cause of cancer death among men and the second leading cause of cancer death among women worldwide. ${ }^{1}$ Surgical resection and chemotherapy are traditional strategies used in the treatment of lung cancer, depending on the disease stage and patient status. However, the results achieved with conventional therapy are unsatisfactory. Recently, the epidermal growth factor receptor-tyrosine kinase inhibitors (EGFR-TKIs) gefitinib, erlotinib, and AZD-9291 ${ }^{2}$ have been used to treat unresectable or recurrent lung cancer. Previous studies have shown that activating mutations in the tyrosine kinase domain of the EGFR gene are associated with sensitivity to EGFR-TKIs. ${ }^{3,4}$ Moreover, EGFR mutations correlate with population characteristics such as nonsmoking status, female sex, East Asian ethnicity, and a diagnosis of adenocarcinoma. ${ }^{4-9}$

Treatment with an EGFR-TKI must be preceded by EGFR testing, whether by Sanger sequencing, allele-specific polymerase chain reaction (PCR), or targeted next- 
generation sequencing. ${ }^{10}$ However, because tissue samples may be unavailable or insufficient, EGFR testing is restricted in clinical practice. Moreover, testing is expensive and, thus, often not feasible, especially in developing countries. It would, therefore, be valuable to be able to predict the curative effect of EGFR-TKIs based on clinicoradiologic characteristics and other easily accessed clinical markers.

In the past decade, several studies relating EGFR mutations to computed tomography (CT) findings in patients with lung cancer have been published. ${ }^{11-16}$ However, to our knowledge, these data have not been used to classify EGFR mutations status with respect to their TKI sensitivity. The ability to distinguish EGFR-TKI-sensitive group vs nonsensitive group may be more significant with respect to clinical decision-making than a classification of EGFR mutation vs wild-type. Furthermore, only few studies have sample sizes large enough to evaluate the correlation between clinical features and EGFR mutation status. Therefore, in a retrospective study of a Chinese cohort of patients, we examined the relationship between EGFR mutation status and clinical characteristics.

\section{Materials and methods Patients}

This study was approved by the Ethical Committee of the First Affiliated Hospital of Wenzhou Medical University as a retrospective study. The requirement for patient informed consent was waived by the Ethical Committee because of the retrospective nature of this study, but patient data confidentiality was protected. Patients who were diagnosed with lung carcinomas and who underwent EGFR testing at the First Affiliated Hospital of Wenzhou Medical University (Wenzhou, Zhejiang, People's Republic of China) between January 1, 2011, and November 31, 2015, were enrolled. Only patients with CT images acquired before the administration of any anticancer therapy, including surgical resection, chemotherapy, radiotherapy, and targeted therapy, were included. The images were retrieved from the institutional Picture Archiving and Communication System. Patients with uncertain EGFR test results, secondary lung cancer, tumors with ambiguous radiological characteristics due to large lobar atelectasis, or endobronchial tumors were excluded.

Thus, 817 patients (467 males and 350 females; age range: 18-88 years) were included in the study (Table 1). Their smoking history was assessed using the Brinkman index, ${ }^{17}$ calculated as the number of cigarettes smoked per day multiplied by the number of smoking years. The histopathological diagnosis was based on the criteria of the WHO/International Association for the Study of Lung Cancer (IASLC) histological classification of lung cancer. ${ }^{18}$ All patients were hospitalized in our institution, where complete clinical data were obtained. The specimens included CT-guided biopsy, bronchoscopic biopsy, pleural effusion, lymph node biopsy, and surgical samples.

\section{EGFR testing}

EGFR mutational analysis was performed on DNA extracted from multisource specimens of formalin-fixed, paraffinembedded lung carcinomas and using a DNA extraction kit

Table I Clinical characteristics of patients in the EGFR-TKI-sensitive group and nonsensitive group

\begin{tabular}{|c|c|c|c|c|}
\hline Characteristics & Nonsensitive group & Sensitive group & Total & $P$-value \\
\hline Number of patients & 455 & 362 & 817 & \\
\hline Age (years) [mean \pm SD] & $64.2 \pm 10.7$ & $63.4 \pm 10.2$ & & $0.24 I$ \\
\hline Sex & & & & $<0.001$ \\
\hline Male & 324 (71.2\%) & 143 (39.5\%) & 467 & \\
\hline Female & $|3|(28.8 \%)$ & $219(60.5 \%)$ & 350 & \\
\hline Smoking index & & & & $<0.001$ \\
\hline$=0$ & $208(45.7 \%)$ & 280 (77.4\%) & 488 & \\
\hline$>0, \leq 500$ & $58(12.8 \%)$ & $40(11.0 \%)$ & 98 & \\
\hline$>500$ & $189(4 \mid .5 \%)$ & 42 (II.6\%) & 231 & \\
\hline Male & & & & $<0.001$ \\
\hline Smoking index $=0$ & 78 (24.1\%) & $61(42.6 \%)$ & 139 & \\
\hline$>0, \leq 500$ & $57(\mathrm{I} 7.6 \%)$ & $40(28.0 \%)$ & 97 & \\
\hline$>500$ & 189 (58.3\%) & $42(29.4 \%)$ & 231 & \\
\hline Nonsmoker & & & & $<0.001$ \\
\hline Male & 78 (37.5\%) & $6 \mathrm{I}(2 \mathrm{I} .8 \%)$ & 139 & \\
\hline Female & $130(62.5 \%)$ & $219(78.2 \%)$ & 349 & \\
\hline
\end{tabular}

Notes: We made a stratified analysis of sex and smoking index, respectively. Because smoking females were in the minority, we only analyzed the above two subgroups. The significant $P$-value is indicated in bold.

Abbreviations: EGFR-TKI, epidermal growth factor receptor-tyrosine kinase inhibitor. 
(Omega Corporation, Yarraville, Victoria, Australia) according to the manufacturer's instructions. The DNA samples were serially diluted to $10 \mathrm{ng} / \mu \mathrm{L}$; their OD 260/280 values were between 1.8 and 2.0.

Twenty-nine EGFR mutations involving exons 18-21 were detected with the Amplified Refractory Mutation System (ARMS), conducted using a DxS EGFR mutation test kit (Amoy Diagnostics, Xiamen, People's Republic of China) according to the manufacturer's instructions. The ARMS analysis is based on a highly sensitive, allele-specific PCR (max. $1 \%$ of mutant DNAs) and is more accurate than DNA direct sequencing. ${ }^{19,20}$ Each PCR tube contained 5-10 pmol specific primers/L, 20 pmol probes/L, $12.5 \mu \mathrm{mol} \mathrm{dNTPs} / \mathrm{L}$, $175 \mu \mathrm{mol}$ magnesium chloride/L, $1 \mathrm{mmol}$ ammonium sulfate $/ \mathrm{L}, 2.5 \mathrm{mmol}$ potassium chloride/L, and $\mathrm{ddH}_{2} \mathrm{O}$. Taq enzyme $(2.7 \mu \mathrm{L})$ was mixed with $42.3 \mu \mathrm{L}$ of $\mathrm{ddH}_{2} \mathrm{O}$, and 5 $\mu \mathrm{L}$ of the mixture was then added to eight real-time PCR tubes. The cycling conditions were as follows: 5 minutes at $95^{\circ} \mathrm{C}, 15$ cycles of $95^{\circ} \mathrm{C}$ for 25 seconds, $64^{\circ} \mathrm{C}$ for 20 seconds, and $72^{\circ} \mathrm{C}$ for 20 seconds, followed by 31 cycles of $93^{\circ} \mathrm{C}$ for 25 seconds, $60^{\circ} \mathrm{C}$ for 35 seconds, and $72^{\circ} \mathrm{C}$ for 20 seconds. Fluorescence was measured at $60^{\circ} \mathrm{C}$.

\section{CT imaging}

A whole-chest CT scan, ranging from the level of the superior aperture of the thorax to the top of the diaphragm, was performed with one breath-hold. The images were reviewed using lung (width: 1,500 HU, level: $-600 \mathrm{HU}$ ) and mediastinal (width: $280 \mathrm{HU}$, level: $35 \mathrm{HU}$ ) window settings. Image reconstruction included three types: $1.25-\mathrm{mm}$ thickness and $1.25-\mathrm{mm}$ interval in 317 (38.8\%) patients, 2-mm thickness and $2-\mathrm{mm}$ interval in $470(57.5 \%)$ patients, and 5-mm thickness and a $5-\mathrm{mm}$ interval in $30(3.7 \%)$ patients.
CT images were independently reviewed by two respiratory physicians (L Yang and Y Lu, First Affiliated Hospital of Wenzhou Medical University) with 10 and 9 years of experience in the interpretation of chest images, respectively. Both were unaware of the EGFR mutation status and pathological result. Differences in their interpretations were resolved by discussion. Tumor size, margin, density, and special signs were evaluated on the CT images.

Tumor size was defined as the maximal diameter of the main nodule and was measured using the measuring tool in the Picture Archiving and Communication System. Tumor density was categorized as pure ground-glass opacity (pGGO), mixed ground-glass opacity (mGGO), and solid patterns according to the CT images. GGO was defined as a hazy increase in lung attenuation on images in which the underlying bronchial or vascular structures were visible. The density was classified on the basis of the GGO ratio, calculated based on the tumor shadow disappearance rate (TDR):21,22

TDR $=1-($ maxD $*$ perD on mediastinal windows/ maxD*perD on lung windows) where maxD is the maximum dimension of the tumor and perD, the largest dimension perpendicular to the maximum axis. ${ }^{12}$ According to the TDR results, three tumor groups ( $\mathrm{pGGO}$, mGGO, and solid) were defined: $\mathrm{TDR}=1, \mathrm{TDR} \geq 0.5$, and TDR $<0.5$, respectively. ${ }^{12}$

The tumor margin was described as smooth, lobulated, spiculated, angular, or obscure (Figure 1), with the main tumor of each patient having margins with one or more characteristics. A smooth margin was smooth and well defined. A lobulated margin was one with at least three relatively obvious undulations in the margin of the mass. Spiculation was defined as the presence of relatively sharp linear projections in the interface between the mass and the lung parenchyma, an angular margin as a sharp triangle - whether

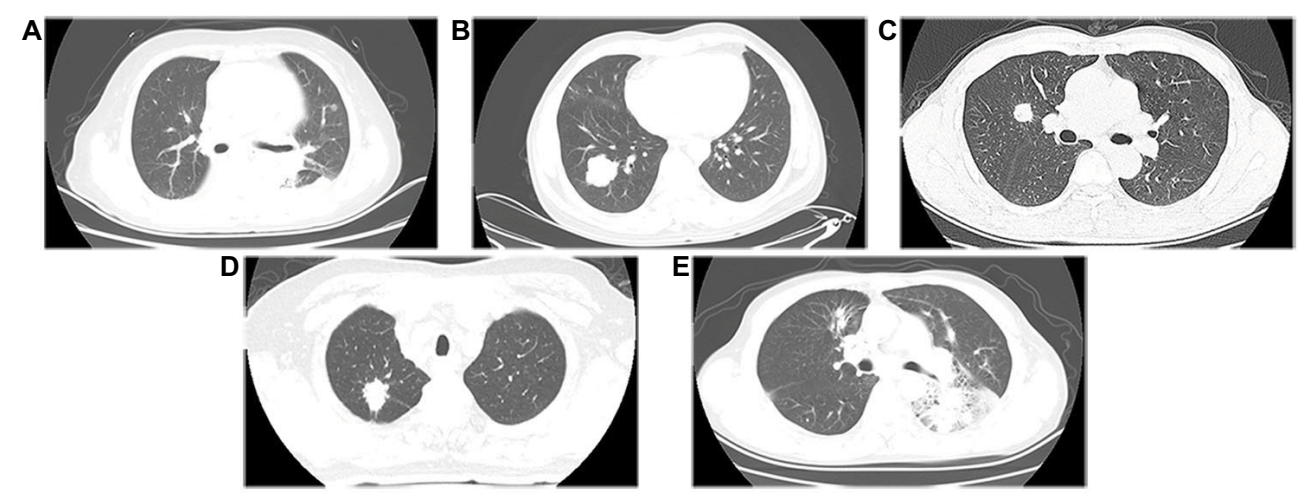

Figure I The five types of tumor margin as seen on the CT images.

Note: (A) smooth; (B) lobulated; (C) spiculated; (D) angular; (E) obscured.

Abbreviation: CT, computed tomography. 
long or short, thick, or thin - projecting from the mass, and an obscured margin as a difficult to distinguish outline of the mass, resembling a tumor surrounded by pneumonia.

The four special signs were those commonly seen on CT images of lung cancer: pleural indentation, air bronchogram, cavitation, and calcification (Figure 2). Pleural indentation was defined as a V-shaped or linear pattern of high attenuation between the lesion and the pleura, due to the traction of cicatricial tissue. An air bronchogram was seen as continuous dots or branching bands of air attenuation within a solid lesion of high attenuation. Cavitation was interpreted as the presence of one or more at least 5-mm round or roundish forms of air attenuation in the lesion, and calcification as the presence of a high-density shadow in the lesion, seen on the mediastinal windows of nonenhanced CT images, and primarily either punctate or sand-like in form.

\section{Statistical analysis}

Numerical variables were evaluated for a normal distribution using the Shapiro-Francia normality test. Differences in the nonnormally distributed variables were compared using a Welch two-sample $t$-test and a Kruskal-Wallis test. Statistical comparisons of ordinal categorical variables were performed using the Mann-Whitney $U$ test and Kruskal-Wallis test. The Pearson $\chi^{2}$ test and the Fisher's exact test were used for unordered categorical variables. A Dunn-Bonferroni approach following the Kruskal-Wallis test was adopted for all pairwise multiple comparisons. The partitions of $\chi^{2}$

A

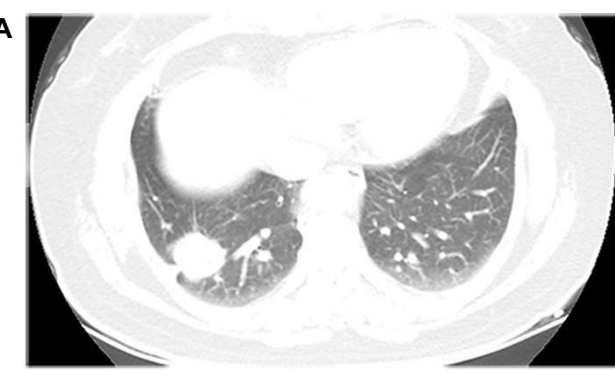

C

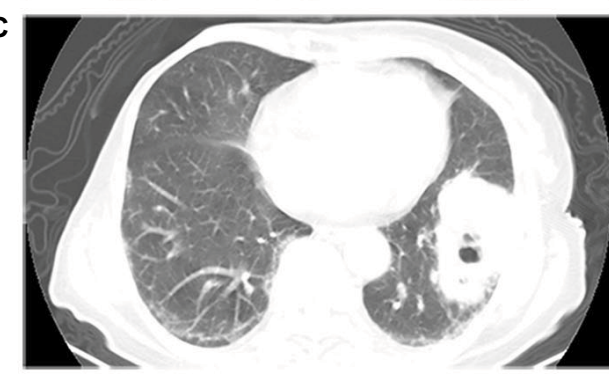

method were used for the Fisher's exact test or the Pearson $\chi^{2}$ test followed by all pairwise multiple comparisons. The adjusted test levels $\left(\alpha^{\prime}\right)$ of multiple comparisons were 0.013 (3 subgroups) and 0.003 (6 subgroups). Logistic regression analyses were performed on the variables representing important CT features. Two-sided $P$-values of $<0.05$ were considered statistically significant. All statistical analyses were performed using SPSS software 20.0 (SPSS Company, Chicago, IL, USA, 2004).

\section{Results}

The results of EGFR testing in 817 patients were as follows: $448(54.8 \%)$ patients were wild-type, $4(0.5 \%)$ patients had a mutation in exon $18(\mathrm{G} 719 \mathrm{X}), 176(21.5 \%)$ had an exon 19 deletion, $7(0.9 \%)$ had a mutation in exon 20 (including insertions in six patients and S768I in one patient), 175 (21.4\%) had a mutation in exon 21 (L858R in 168 patients and L861Q in seven patients), and seven patients ( $0.9 \%)$ had compound mutations (exon 19 deletion/L858R in one patient, G719X/S768I in two patients, exon 19 deletion/T790M in one patient, and G719X/L861Q in three patients). EGFR-TKInonsensitive group was made up of those with the wild-type, in patients with an exon 20 insertion, and in the patient with an exon 19 deletion/T790M compound mutation. The other mutation subtypes were classified as EGFR-TKI-sensitive group. $^{23-26}$

The clinical characteristics of the patients are shown in Tables 1 and 2. The 467 males and 350 females included
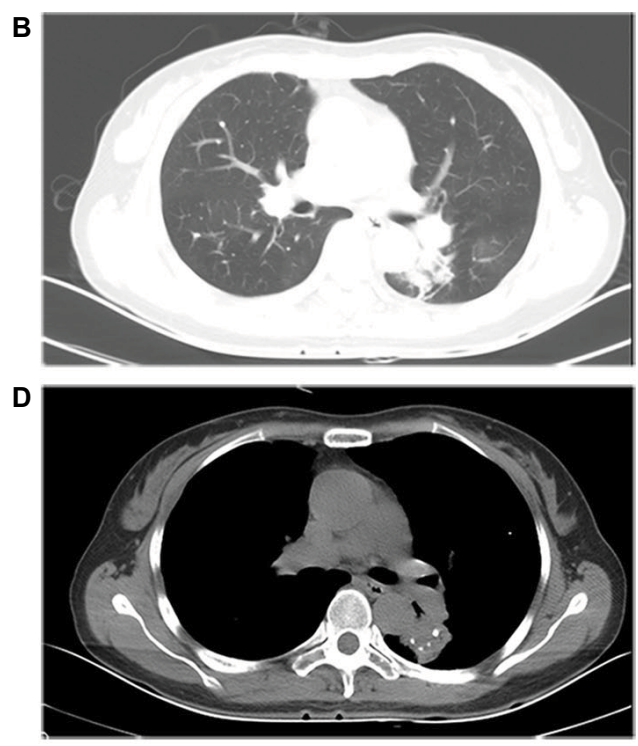

Figure 2 The four special signs seen on the CT images.

Note: (A) pleural indentation; (B) air bronchogram; (C) cavitation; (D) calcification. Abbreviation: CT, computed tomography. 
in our study had a median age of 64.0 years (range: $18-88$ years). The smoking index data were divided into three groups $(0 ;>0, \leq 500 ;>500)$, comprising 488, 98, and 231 of the patients, respectively. Female patients and patients with a low smoking index had a significantly higher rate of sensitive as opposed to nonsensitive group ( $P<0.001$, both). Patients in the sensitive group did not significantly differ in age from those in the nonsensitive group $(P=0.241)$. Multiple comparisons of mutation subtypes showed that female sex was significantly related to lung carcinomas carrying an exon 19 deletion $(P<0.001)$ or an exon 21 mutation $(P<0.001)$ rather than those in which a wild-type EGFR was detected. In patients with a low smoking index, EGFRs with an exon 19 deletion $(P<0.001)$ or exon 21 mutation $(P<0.001)$ occurred significantly more often than did the wild-type. Age was not statistically significant among different mutation subtypes based on multiple comparisons following the Kruskal-Wallis test. A stratified analysis of sex and smoking index was also performed. Among males, those with a low smoking index had a significantly higher rate of sensitive as opposed to nonsensitive group $(P<0.001)$. In the nonsmoking (smoking index $=0$ ) group, females had a significantly higher rate of sensitive as opposed to nonsensitive group $(P<0.001)$.

The CT features of patients in the EGFR-TKI-sensitive and nonsensitive group are presented in Table 3 . The mean tumor diameters were $37.1 \pm 18.8 \mathrm{~mm}$ (range: 6-114 $\mathrm{mm}$ ) and $43.3 \pm 23.2 \mathrm{~mm}$ (range: $3-140 \mathrm{~mm}$ ), respectively. After correcting for age, sex, and histological type, the results of logistic regression analysis identified tumor size $(P<0.001$, odds ratio $[\mathrm{OR}]=0.984,95 \% \mathrm{CI}=0.976-0.992)$, smooth margins $(P=0.015, \mathrm{OR}=0.341,95 \% \mathrm{CI}=0.144-0.810)$, and angular margins $(P<0.001, \mathrm{OR}=0.544,95 \% \mathrm{CI}=0.393-0.754)$ as independent negative predictors of EGFR-TKI-sensitive group. Pleural indentation $(P<0.001, \mathrm{OR}=1.969,95 \% \mathrm{CI}$ $=1.391-2.788)$ and air bronchogram $(P=0.025, \mathrm{OR}=1.484$, $95 \% \mathrm{CI}=1.051-2.095)$ were independent positive predictors of EGFR-TKI-sensitive group, whereas lobulated margin, spiculated margin, obscured margin, cavitation, and calcification were not.

The CT features of the patients according to EGFR mutation subtype are shown in Figure 3 and Table 4. Multiple comparisons of mutation subtypes showed that tumors with exon 21 mutation were significantly smaller than those with wild-type EGFR $(P<0.001)$; pleural indentation was significantly more common in the exon 21 mutation group than in the wild-type group $(P=0.002)$; air bronchogram was significantly more common in the exon 19 deletion group than in either the wild-type group $(P=0.001)$ or the exon 21 mutation group $(P=0.026)$. Among the various EGFR mutation subtypes, there were no significant differences with respect to tumor density, any type of margin, cavitation, or calcification.

Table 3 CT features of patients with EGFR-TKI-sensitive group and nonsensitive group

\begin{tabular}{|l|l|l|l|}
\hline Independent variable & $\mathbf{P}$-value & $\mathbf{O R}$ & $\mathbf{9 5} \% \mathbf{C l}$ \\
\hline Tumor size $(\mathrm{mm})$ & $<\mathbf{0 . 0 0 I}$ & 0.984 & $0.976-0.992$ \\
\hline Margin & & & \\
\hline Smooth & $\mathbf{0 . 0 I 5}$ & $0.34 \mathrm{I}$ & $0.144-0.8 \mathrm{I} 0$ \\
\hline Angle & $<\mathbf{0 . 0 0 I}$ & 0.544 & $0.393-0.754$ \\
\hline Special signs & & & \\
\hline Pleural indentation & $<\mathbf{0 . 0 0 I}$ & $\mathbf{I} .969$ & $\mathrm{I} .39 \mathrm{I}-2.788$ \\
\hline Air bronchogram & $\mathbf{0 . 0 2 5}$ & $\mathrm{I} .484$ & $\mathrm{I} .05 \mathrm{I}-2.095$ \\
\hline
\end{tabular}

Note: The significant $P$-value is indicated in bold.

Abbreviations: CT, computed tomography; EGFR-TKI, epidermal growth factor receptor-tyrosine kinase inhibitor; OR, odds ratio.

Table 2 Clinical characteristics of patients with EGFR mutation subtypes

\begin{tabular}{|c|c|c|c|c|c|c|c|c|}
\hline Characteristics & Wild-tpye & $\begin{array}{l}\text { Exon } 18 \\
\text { point } \\
\text { mutation }\end{array}$ & $\begin{array}{l}\text { Exon } 19 \\
\text { deletion }\end{array}$ & $\begin{array}{l}\text { Exon } 20 \\
\text { mutation }\end{array}$ & $\begin{array}{l}\text { Exon } 2 \text { I } \\
\text { mutation }\end{array}$ & $\begin{array}{l}\text { Compound } \\
\text { mutation }\end{array}$ & Total & $P$-value \\
\hline Number of patients & 448 & 4 & 176 & 7 & 175 & 7 & 817 & \\
\hline Age (years) $[$ mean $\pm S D]$ & $64.3 \pm 10.7$ & $69.8 \pm 8.3$ & $61.8 \pm 10.0$ & $59.0 \pm 11.0$ & $64.6 \pm 10.1$ & $68.0 \pm 10.5$ & & 0.032 \\
\hline Sex & & & & & & & & $<0.001$ \\
\hline Male & $32 ।(71.7 \%)$ & $2(50.0 \%)$ & $66(37.5 \%)$ & $4(57.1 \%)$ & $73(41.7 \%)$ & I (I4.3\%) & 467 & \\
\hline Female & $127(28.3 \%)$ & $2(50.0 \%)$ & $110(62.5 \%)$ & $3(42.9 \%)$ & $102(58.3 \%)$ & $6(85.7 \%)$ & 350 & \\
\hline Smoking index & & & & & & & & $<0.001$ \\
\hline$\leq 0$ & 204 (45.5\%) & $2(50.0 \%)$ & I 37 (77.8\%) & $3(42.9 \%)$ & $136(77.7 \%)$ & $6(85.7 \%)$ & 488 & \\
\hline$>0, \leq 500$ & $57(12.7 \%)$ & $2(50.0 \%)$ & $20(11.4 \%)$ & $2(28.6 \%)$ & 17 (9.7\%) & $0(0.0 \%)$ & 98 & \\
\hline$>500$ & $187(4 \mid .7 \%)$ & $0(0.0 \%)$ & $19(10.8 \%)$ & $2(28.6 \%)$ & $22(12.6 \%)$ & I (I4.3\%) & 231 & \\
\hline
\end{tabular}

Note: The significant $P$-value is indicated in bold.

Abbreviation: EGFR, epidermal growth factor receptor. 
The histological classification of the tumors with respect to EGFR-TKI-sensitive and nonsensitive groups is presented in Table 5. Among the three histological types (adenocarcinoma, squamous cell carcinoma, and adenosquamous carcinoma), patients with squamous cell carcinoma had significantly fewer sensitive mutations than those with either adenocarcinoma $(P<0.001)$ or adenosquamous carcinoma $(P<0.001)$. Of the remaining five histological types (largecell carcinoma, neuroendocrine carcinoma, sarcomatoid carcinoma, lymphoepithelioma, and mucoepidermoid carcinoma), there were only two cases of sensitive mutations, one occurring in a sarcomatoid carcinoma (1/4, 25\%) and the other in a mucoepidermoid carcinoma $(1 / 1,100 \%)$.

\section{Discussion}

EGFR-TKIs significantly improve progression-free survival in patients with EGFR-TKI-sensitive mutations, ${ }^{27,28}$ whereas, similar to tumors carrying the wild-type EGFR, those with drug-resistant mutations (exon 20 insertion and T790M mutation) are insensitive to EGFR-TKIs. Therefore, the

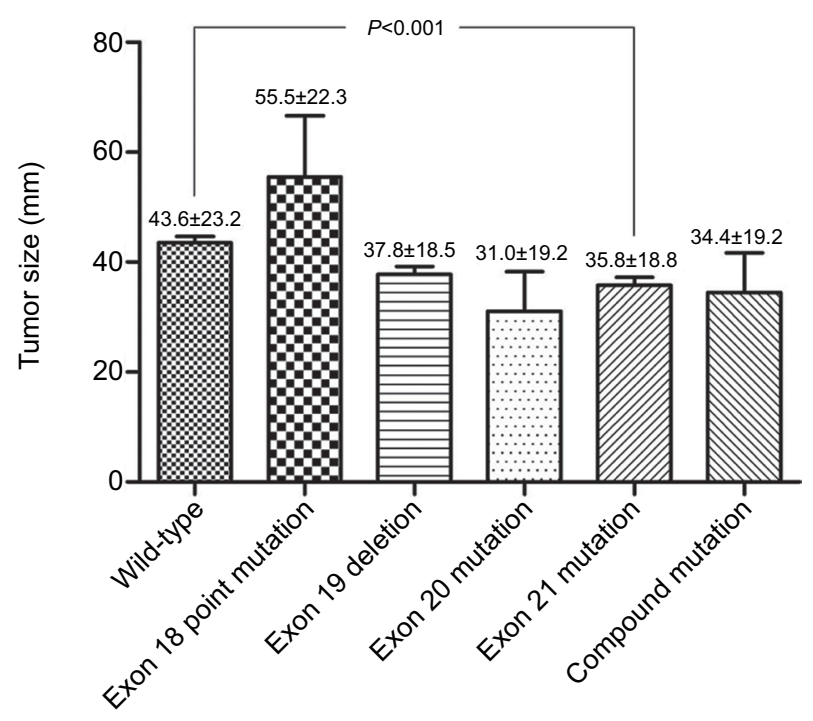

Figure 3 Relationship between tumor size and EGFR mutation subtypes. Abbreviation: EGFR, epidermal growth factor receptor. classification method used in this study (sensitive and nonsensitive group) enables a more rational approach to clinical drug selection when tissue samples are either not available or insufficient. In this study, the rate of EGFR-TKI-sensitive mutations, as determined in an ARMS analysis, was 44.3\% $(362 / 817)$, which was not completely consistent with the results of several other studies in which an ARMS analysis of Asian patients was also performed. ${ }^{14,29}$ The main reason for the differences between our study and the others can be attributed to differences in the ratio of females to males in the study population.

The vast majority of females in our study did not have a smoking habit. A stratified analysis showed that female sex and a low smoking index were independently associated with EGFR-TKI-sensitive mutations, as also reported in previous studies. ${ }^{29}$ In addition, exon 19 deletion and exon 21 mutation were more frequent than wild-type EGFR in the lung tumors of females and patients with a low smoking index, as also shown in other studies. ${ }^{12,15,30}$ The reason for the greater frequency of these mutations in females is unknown but may be related to estrogen levels and estrogen receptor expression. ${ }^{31,32}$ Smoking correlates with EGFR mutations and, to some extent, accounts for their incidence. Khan et $\mathrm{al}^{33}$ showed that cigarette smoke produces $\mathrm{H}_{2} \mathrm{O}_{2}$-induced oxidative stress, which in turn aberrantly activates the EGFR. Goldkorn Filosto's ${ }^{34}$ study systematically demonstrated that nSMase2, activated by cigarette smoke, was fatal for exosome generation and, thereby, played a role in the spread of activated oncogenes (such as EGFR) and miRNA, leading to tumorigenesis. Further studies are needed to elucidate the mechanism linking these events.

Our study also examined the relationship between EGFRTKI-sensitive mutations and CT features, showing that tumor density was not an independent predictor of these mutations. However, both mGGO and pGGO are common CT features of early-stage lung cancer. Because many of our study patients had advanced-stage disease, $\mathrm{mGGO}$ and $\mathrm{pGGO}$ were seen in only a few samples and their relationship to EGFR mutations

Table 4 CT features of patients with EGFR mutation subtypes

\begin{tabular}{|c|c|c|c|c|c|c|c|c|}
\hline $\begin{array}{l}\text { Special signs of } \\
\text { CT images }\end{array}$ & Wild-type & $\begin{array}{l}\text { Exon I } 8 \\
\text { point } \\
\text { mutation }\end{array}$ & $\begin{array}{l}\text { Exon } 19 \\
\text { deletion }\end{array}$ & $\begin{array}{l}\text { Exon } 20 \\
\text { mutation }\end{array}$ & $\begin{array}{l}\text { Exon 21 } \\
\text { mutation }\end{array}$ & $\begin{array}{l}\text { Compound } \\
\text { mutation }\end{array}$ & Total & $P$-value \\
\hline Number of patients & 448 & 4 & 176 & 7 & 175 & 7 & 817 & \\
\hline Pleural indentation & 267 (50.66\%) & $4(0.76 \%)$ & $123(23.34 \%)$ & $3(0.57 \%)$ & $128(24.29 \%)$ & $2(0.38 \%)$ & 527 & $<0.001$ \\
\hline Air bronchogram & I 48 (50.68\%) & $3(1.03 \%)$ & $83(28.42 \%)$ & $2(0.68 \%)$ & $55(18.84 \%)$ & I (0.34\%) & 292 & 0.003 \\
\hline Cavitation & $52(63.41 \%)$ & $0(0.00 \%)$ & 15 (I8.29\%) & $0(0.00 \%)$ & $15(18.29 \%)$ & $0(0.00 \%)$ & 82 & 0.756 \\
\hline Calcification & 7I (63.96\%) & $0(0.00 \%)$ & $2 \mathrm{I}(18.92 \%)$ & 2 (1.80\%) & 16 (14.41\%) & I (0.90\%) & III & 0.151 \\
\hline
\end{tabular}

Note: The significant $P$-value is indicated in bold.

Abbreviations: CT, computed tomography; EGFR, epidermal growth factor receptor. 
Table 5 Histological classifications in the EGFR-TKI-sensitive group and nonsensitive group

\begin{tabular}{|l|l|l|l|l|}
\hline Histological classifications & Nonsensitive group & Sensitive group & Total & P-value \\
\hline Number of patients & 455 & 362 & 817 & $<\mathbf{0 . 0 0}^{\mathrm{a}}$ \\
\hline Adenocarcinoma & $323(7 \mathrm{I} .0 \%)$ & $337(93.1 \%)$ & 660 & \\
\hline Squamous-cell carcinoma & $\mathrm{I} 05(23.1 \%)$ & $\mathrm{I}(3.3 \%)$ & $\mathrm{II}$ & \\
\hline Adenosquamous carcinoma & $\mathrm{II}(2.4 \%)$ & $\mathrm{II}(3.0 \%)$ & 22 & \\
\hline Large-cell carcinoma & $2(0.4 \%)$ & $0(0.0 \%)$ & 2 & \\
\hline Neuroendocrine carcinoma & $9(2.0 \%)$ & $0(0.0 \%)$ & 9 & \\
\hline Sarcomatoid carcinoma & $3(0.7 \%)$ & $\mathrm{I}(0.3 \%)$ & 4 & \\
\hline Lymphoepithelioma & $2(0.4 \%)$ & $0(0.0 \%)$ & 2 & \\
\hline Mucoepidermoid carcinoma & $0(0.0 \%)$ & $\mathrm{I}(0.3 \%)$ & $\mathrm{I}$ & \\
\hline
\end{tabular}

Notes: ${ }^{P}$-value was based on comparison between three histological types (adenocarcinoma, squamous-cell carcinoma and adenosquamous carcinoma). The significant $P$-value is indicated in bold.

Abbreviation: EGFR-TKI, epidermal growth factor receptor-tyrosine kinase inhibitor.

remains to be determined. In our study, the tumor diameter was an independent negative predictor of EGFR-TKI-sensitive mutations, and tumors with exon 21 mutation were smaller than those carrying wild-type EGFR. Previous studies reported a tendency of tumors with wild-type EGFR to be larger on CT scans than those with mutated EGFRs, which supported our results to some extent. ${ }^{11,16,35}$ However, this possible relationship does not further our understanding of the EGFR as an oncogene and, thus, its involvement in tumor enhancement. ${ }^{36}$

In addition to tumor size, we assessed the tumor margins as a function of EGFR mutation status. The results revealed that both smooth and angular margins were independent negative predictors of EGFR-TKI-sensitive mutations. However, as the first report of this association, it must still be verified in further studies. It may be that EGFR gene status affects the tumor's growth pattern in terms of an uneven growth rate at the tumor periphery, with growth resistance and the tumor blood supply combining to produce angular margins.

Both pleural indentation and air bronchogram were identified as independent positive predictors of EGFR-TKIsensitive mutations. The pattern of pleural indentation, an important imaging characteristic in the diagnosis of lung cancer, is determined by reactive fibrosis and cicatrization of the tumor. Pleural indentation is a common feature of adenocarcinoma but is not seen in the majority of squamous cell carcinomas, ${ }^{37}$ which would perhaps explain why pleural indentation occurred more often in the group with sensitive mutations. The relationship between pleural indentation and EGFR mutation status has, to the best of our knowledge, not been previously reported. Air bronchogram is a characteristic feature of bronchioloalveolar carcinoma (BAC). Consistent with our results, a positive correlation between EGFR mutations and adenocarcinoma with BAC components was previously reported. ${ }^{38}$ The new international multidisciplinary histological classification of lung adenocarcinoma, published by the IASLC/American Thoracic Society (ATS)/European Respiratory Society (ERS) in 2011, abolished the concept of BAC because its features can occur in many subtypes of adenocarcinoma. ${ }^{39}$ The new IASLC/ATS/ERS classification may be more suitable for future research.

The presence of calcification is an important factor in assessing the nature of a lung nodule. CT offers better density resolution than conventional chest imaging and has led to an estimated prevalence of calcification in lung cancer as high as $5 \%-10 \%{ }^{40,41}$ Calcification in lung cancer may be primary and engulfed by the tumor, occur within areas of tumor necrosis, or manifest as calcium deposition following calcium secretion by the carcinoma itself. Cao et $\mathrm{a}^{42}$ demonstrated that the sign of calcification in CT images showed no statistical significance between wild-type group and mutated group, which supported our results. However, another study found that calcification was an independent negative predictor of EGFR mutations and that, in adenocarcinoma, EGFR mutation is vital to the pathogenesis of nonmucinous but not mucinous BAC-type tumors, with the former leading to calcium deposition. ${ }^{16}$ Calcified tumors also tend to be larger, ${ }^{43}$ which may explain their relationship to the EGFR, but the exact mechanism remains to be elucidated.

In evaluating the correlation between EGFR mutation status and histological classification, our study showed that patients with squamous cell carcinoma had fewer sensitive mutations than those with adenocarcinoma or adenosquamous carcinoma. Thus, tumors with an adenocarcinoma component correlated with EGFR-TKI-sensitive mutations. These results are consistent with previous reports. ${ }^{29}$ However, most of those studies focused on adenocarcinoma, whereas much less is known about the correlations between EGFR mutation status and the clinical features of nonadenocarcinoma. In their study of Chinese patients with lung squamous carcinoma, Memon et $\mathrm{a}^{44}$ found that among EGFR-mutation-positive patients, $88.3 \%$ had EGFR-TKI-sensitive mutations. This 
rate is much higher than the rate in our study, perhaps due to the different methods adopted. In their study, two types of deletion and six point mutations were detected in EGFR exon 19 by PCR-denature gel gradient electrophoresis, which, with its higher sensitivity, may be useful in future investigations.

Our study also had several limitations. First, the specimens used for EGFR testing were not from a single, ample surgical sample, which would have minimized the risk of false-negative results in EGFR testing. However, in most cases, genomic DNA extracted from nonoperative biopsy tissue is sufficient for EGFR testing in an ARMS analysis. Second, we did not calculate tumor volume to evaluate the GGO component within the tumor, although the TDR method is appropriate to evaluate the GGO proportion. ${ }^{45}$ Third, the absence of evaluating the predictive role of enhanced-CT is also a limitation in our current work. Fourth, with its retrospective design, our study may have been vulnerable to sample selection bias because the vast majority of lung cancer patients seen at our hospital have late-stage disease. Prospective studies are needed to confirm our findings.

\section{Conclusion}

According to our study, clinical information, including sex, smoking index, and CT features, may be useful in guiding therapeutic decision-making when EGFR testing is not feasible. The identification of other informative and readily available indicators is needed to predict the efficacy of EGFR-TKI treatment.

\section{Acknowledgment}

We thank the two respiratory physicians (L Yang and Y $\mathrm{Lu}$ ) for CT image interpretation, and the staff of the record retrieval department from the First Affiliated Hospital of Wenzhou Medical University for their efforts.

\section{Author contribution}

WZ conceived and designed the study; XQ, XG, and WZ provided the study materials and aided in patient participation; XQ, XG, and WZ helped with the collection and assembly of data. All authors contributed toward data analysis, drafting and critically revising the paper and agree to be accountable for all aspects of the work.

\section{Disclosure}

The authors report no conflicts of interest in this work.

\section{References}

1. Torre LA, Siegel RL, Jemal A. Lung Cancer Statistics.. Adv Exp Med Biol. 2016;893:1-19.
2. Jänne PA, Yang JC, Kim DW, et al. AZD9291 in EGFR inhibitor-resistant non-small-cell lung cancer. N Engl J Med. 2015;372(18):1689-1699.

3. Pao W, Miller V, Zakowski M, et al. EGF receptor gene mutations are common in lung cancers from "never smokers" and are associated with sensitivity of tumors to gefitinib and erlotinib. Proc Natl Acad Sci US A. 2004;101(36):13306-13311.

4. Paez JG, Jänne PA, Lee JC, et al. EGFR mutations in lung cancer: correlation with clinical response to gefitinib therapy. Science. 2004;304(5676):1497-1500.

5. Gazdar AF. Personalized medicine and inhibition of EGFR signaling in lung cancer. N Engl J Med. 2009;361(10):1018-1020.

6. Lynch TJ, Bell DW, Sordella R, et al. Activating mutations in the epidermal growth factor receptor underlying responsiveness of non-small-cell lung cancer to gefitinib. N Engl J Med. 2004;350(21):2129-2139.

7. Kosaka T, Yatabe Y, Endoh H, Kuwano H, Takahashi T, Mitsudomi T. Mutations of the epidermal growth factor receptor gene in lung cancer: biological and clinical implications. Cancer Res. 2004;64(24): 8919-8923.

8. Mitsudomi T, Kosaka T, Yatabe Y. Biological and clinical implications of EGFR mutations in lung cancer. Int J Clin Oncol. 2006;11(3):190-198.

9. Shigematsu H, Lin L, Takahashi T, et al. Clinical and biological features associated with epidermal growth factor receptor gene mutations in lung cancers. J Natl Cancer Inst. 2005;97(5):339-346.

10. Sheikine Y, Rangachari D, Mcdonald DC, et al. EGFR Testing in Advanced Non-Small-Cell Lung Cancer, A Mini-Review. Clin Lung Cancer. 2016;17(6):483-492.

11. Yano M, Sasaki H, Kobayashi Y, et al. Epidermal growth factor receptor gene mutation and computed tomographic findings in peripheral pulmonary adenocarcinoma. J Thorac Oncol. 2006;1(5):413-416.

12. Hsu KH, Chen KC, Yang TY, et al. Epidermal growth factor receptor mutation status in stage I lung adenocarcinoma with different image patterns. J Thorac Oncol. 2011;6(6):1066-1072.

13. Tomita M, Ayabe T, Chosa E, Kawagoe K, Nakamura K. Epidermal growth factor receptor mutations in Japanese men with lung adenocarcinomas. Asian Pac J Cancer Prev. 2014;15(24):10627-10630.

14. Yang Y, Yang Y, Zhou X, et al. EGFR L858R mutation is associated with lung adenocarcinoma patients with dominant ground-glass opacity. Lung Cancer. 2015;87(3):272-277.

15. Lee HJ, Kim YT, Kang CH, et al. Epidermal growth factor receptor mutation in lung adenocarcinomas: relationship with CT characteristics and histologic subtypes. Radiology. 2013;268(1):254-264.

16. Hsu JS, Huang MS, Chen CY, et al. Correlation between EGFR mutation status and computed tomography features in patients with advanced pulmonary adenocarcinoma. J Thorac Imaging. 2014;29(6):357-363.

17. Brinkman GL, Coates EO. The effect of bronchitis, smoking, and occupation on ventilation. Am Rev Respir Dis. 1963;87:684-693.

18. Yang X, Lin D. Changes of 2015 WHO Histological Classification of Lung Cancer and the Clinical Significance. Zhongguo Fei Ai Za Zhi. 2016;19(6):332-336.

19. Kimura H, Fujiwara Y, Sone T, et al. High sensitivity detection of epidermal growth factor receptor mutations in the pleural effusion of non-small cell lung cancer patients. Cancer Sci. 2006;97(7):642-648.

20. Ellison G, Donald E, McWalter G, et al. A comparison of ARMS and DNA sequencing for mutation analysis in clinical biopsy samples. $J$ Exp Clin Cancer Res. 2010;29:132.

21. Takamochi K, Nagai K, Yoshida J, et al. Pathologic N0 status in pulmonary adenocarcinoma is predictable by combining serum carcinoembryonic antigen level and computed tomographic findings. JThorac Cardiovasc Surg. 2001;122(2):325-330.

22. Ikehara M, Saito H, Kondo T, et al. Comparison of thin-section CT and pathological findings in small solid-density type pulmonary adenocarcinoma: prognostic factors from CT findings. Eur J Radiol. 2012;81(1):189-194.

23. Chiu CH, Yang CT, Shih JY, et al. Epidermal Growth Factor Receptor Tyrosine Kinase Inhibitor Treatment Response in Advanced Lung Adenocarcinomas with G719X/L861Q/S768I Mutations. $J$ Thorac Oncol. 2015;10(5):793-799. 
24. Banno E, Togashi Y, Nakamura Y, et al. Sensitivities to various epidermal growth factor receptor-tyrosine kinase inhibitors of uncommon epidermal growth factor receptor mutations L861Q and S768I: What is the optimal epidermal growth factor receptor-tyrosine kinase inhibitor? Cancer Sci. 2016;107(8):1134-1140.

25. $\mathrm{Xu}$ J, Jin B, Chu T, et al. EGFR tyrosine kinase inhibitor (TKI) in patients with advanced non-small cell lung cancer (NSCLC) harboring uncommon EGFR mutations: A real-world study in China. Lung Cancer. 2016;96:87-92.

26. Kobayashi Y, Mitsudomi T. Not all epidermal growth factor receptor mutations in lung cancer are created equal: Perspectives for individualized treatment strategy. Cancer Sci. 2016;107(9):1179-1186.

27. Mok TS, WuYL, Thongprasert S, et al. Gefitinib or carboplatin-paclitaxel in pulmonary adenocarcinoma. N Engl J Med. 2009;361(10):947-957.

28. Zhou C, Wu YL, Chen G, et al. Erlotinib versus chemotherapy as first-line treatment for patients with advanced EGFR mutationpositive non-small-cell lung cancer (OPTIMAL, CTONG-0802): a multicentre, open-label, randomised, phase 3 study. Lancet Oncol. 2011;12(8):735-742.

29. Lai Y, Zhang Z, Li J, et al. EGFR mutations in surgically resected fresh specimens from 697 consecutive Chinese patients with non-small cell lung cancer and their relationships with clinical features. Int J Mol Sci. 2013;14(12):24549-24559.

30. Lee YJ, Shim HS, Kang YA, et al. Dose effect of cigarette smoking on frequency and spectrum of epidermal growth factor receptor gene mutations in Korean patients with non-small cell lung cancer. J Cancer Res Clin Oncol. 2010;136(12):1937-1944.

31. Siegfried JM. Women and lung cancer: does oestrogen play a role? Lancet Oncol. 2001;2(8):506-513.

32. He Q, Zhang M, Zhang J, et al. Correlation between epidermal growth factor receptor mutations and nuclear expression of female hormone receptors in non-small cell lung cancer: a meta-analysis. J Thorac Dis. 2015;7(9):1588-1594.

33. Khan EM, Lanir R, Danielson AR, Goldkorn T. Epidermal growth factor receptor exposed to cigarette smoke is aberrantly activated and undergoes perinuclear trafficking. Faseb J. 2008;22(3):910-917.
34. Goldkorn T, Filosto S. Lung injury and cancer: Mechanistic insights into ceramide and EGFR signaling under cigarette smoke. Am J Respir Cell Mol Biol. 2010;43(3):259-268.

35. Usuda K, Sagawa M, Motono N, et al. Relationships between EGFR mutation status of lung cancer and preoperative factors - are they predictive? Asian Pac J Cancer Prev. 2014;15(2):657-662.

36. Doroshow JH. Targeting EGFR in non-small-cell lung cancer. $N$ Engl J Med. 2005;353(2):200-202.

37. Sone S, Sakai F, Takashima S, et al. Factors affecting the radiologic appearance of peripheral bronchogenic carcinomas. JThorac Imaging. 1997;12(3):159-172.

38. Haneda H, Sasaki H, Lindeman N, et al. A correlation between EGFR gene mutation status and bronchioloalveolar carcinoma features in Japanese patients with adenocarcinoma. Jpn J Clin Oncol. 2006;36(2):69-75.

39. Travis WD, Brambilla E, Noguchi M, et al. International association for the study of lung cancer/american thoracic society/european respiratory society international multidisciplinary classification of lung adenocarcinoma. J Thorac Oncol. 2011;6(2):244-285.

40. Okimoto N, Asaoka N, Fujita K. Computed tomography of lung cancer: frequency and characteristics of calcification. Intern Med. 2003;42(9):906-907.

41. Khan AN, Al-Jahdali HH, Allen CM, Irion KL, Al Ghanem S, Koteyar SS. The calcified lung nodule: What does it mean? Ann Thorac Med. 2010;5(2):67-79.

42. Cao Y, Xu H. A new predictive scoring system based on clinical data and computed tomography features for diagnosing EGFR-mutated lung adenocarcinoma. Curr Oncol. 2018;25(2):132-e138.

43. Grewal RG, Austin JH. CT demonstration of calcification in carcinoma of the lung. J Comput Assist Tomogr. 1994;18(6):867-871.

44. Memon AA, Zhang H, Gu Y, et al. EGFR with TKI-sensitive mutations in exon 19 is highly expressed and frequently detected in Chinese patients with lung squamous carcinoma. Onco Targets Ther. 2017;10:4607-4613.

45. Okada M, Nishio W, Sakamoto T, Uchino K, Tsubota N. Discrepancy of computed tomographic image between lung and mediastinal windows as a prognostic implication in small lung adenocarcinoma. Ann Thorac Surg. 2003;76(6):1828-1832.
Cancer Management and Research

\section{Publish your work in this journal}

Cancer Management and Research is an international, peer-reviewed open access journal focusing on cancer research and the optimal use of preventative and integrated treatment interventions to achieve improved outcomes, enhanced survival and quality of life for the cancer patient. The manuscript management system is completely online and includes

\section{Dovepress}

a very quick and fair peer-review system, which is all easy to use. Visit http://www.dovepress.com/testimonials.php to read real quotes from published authors. 